
\title{
28 Research Square \\ CEND1 and GJB6 co-expression as a prognostic factor in colorectal cancer
}

\section{Rui Chen ( $\square$ chenruiwz@zju.edu.cn )}

Department of Neonatal Surgery, children's Hospital Affiliated to Medical College of Zhejiang University, National Center for clinical medicine of children's health and disease. Hang Zhou, Zhe Jiang.

\section{Zhan Wang}

Department of Neonatal Surgery, children's Hospital Affiliated to Medical College of Zhejiang University, National Center for clinical medicine of children's health and disease. Hang Zhou, Zhe Jiang.

\section{Dong Ma}

Department of Neonatal Surgery, children's Hospital Affiliated to Medical College of Zhejiang University, National Center for clinical medicine of children's health and disease. Hang Zhou, Zhe Jiang.

\section{Yijiang Han}

Department of Neonatal Surgery, children's Hospital Affiliated to Medical College of Zhejiang University, National Center for clinical medicine of children's health and disease. Hang Zhou, Zhe Jiang.

\section{Junsong Liu}

Department of General Surgery, The First Affiliated Hospital, College of Medicine, Xi'an Jiaotong University, Xi'an, Shaanxi

\section{Xiangming Che}

Department of General Surgery, The First Affiliated Hospital, College of Medicine, Xi'an Jiaotong University, Xi'an, Shaanxi

\section{Research Article}

Keywords: colorectal cancer, CEND1, GJB6, prognosis

Posted Date: July 13th, 2021

DOl: https://doi.org/10.21203/rs.3.rs-709485/v1

License: (c) (1) This work is licensed under a Creative Commons Attribution 4.0 International License. Read Full License 


\section{Abstract}

Background: Perineural invasion is an important mechanism of cancer progression that is not well understood at present. The present study explored the relationship between GJB6, CEND1, and cell-cell communication as regulators of colorectal cancer patient survival and clinicopathological findings.

Method: Immunohistochemical staining was performed to assess CEND1 and GJB6 expression levels in CRC patient samples, while survival outcomes were assessed using Kaplan-Meier curves and log-rank tests.

Results: Elevated CEND1 expression was associated with tumor location, poor differentiation, and perineural invasion, while GJB6 expression was positively correlated with TNM stage, distant metastasis, and perineural invasion. In addition, GJB6 and CEND1 protein levels were correlated with one another in CRC patient tissues, and high expression of both of these proteins was associated with a higher risk of perineural invasion. CEND1+/GJB6+ status was also associated with poorer patient survival, highlighting both of these proteins as prognostic biomarkers in CRC patients.

Conclusion: Elevated levels of CEND1 and GJB6 are independent predictors of poorer CRC patient prognosis.

\section{Introduction}

Colorectal cancer (CRC) is among the leading causes of cancer-related mortality globally[1]. Aberrant neuronal distribution can facilitate tumor progression, as tumors can spread via perineural invasion. However, the specific role of neurons in the context of cancer progression is not well understood, particularly in the context of CRC. While local CRC patients have a 5 -year survival rate of almost $90 \%$, this rate falls to just $64 \%$ in those exhibiting perineural invasion[2]. CRC is currently treated via a combination of radical surgery and adjuvant chemotherapy or related approaches[3, 4]. Some recent evidence suggests that the innervation of tumors can facilitate their growth. As such, it is vital that the molecular mechanisms governing CRC perineural invasion be clarified[5-8]. To that end, we have focused on identifying novel biomarkers of nerve infiltration associated with this cancer type.

Many different neuron-related proteins have been identified in cancer tissues. For example, CEND1 (Cell Cycle Exit and Neuronal Differentiation 1) is a gene encoding the BM88 protein that is specific to neuronal lineage cells wherein it regulates cell cycle exit and differentiation[9, 10]. As such, this gene may play a role in the development of perineural invasion in certain cancer types[11, 12]. The development of such invasion is inextricably linked to cell-cell contact. Gap junctions form as membrane channels which facilitate small molecule transfer between neighboring cells, regulating processes such as cell cycle progression, signal transduction, and invasion. These junctions are formed by different connexin proteins, of which the isotypes GJA1 (connexin 43) [13, 14]and GJB2 (connexin 26) $[15,16]$ are the best studied. Both GJA1 and GJB2 are frequently upregulated in tumors, wherein they are associated with invasion, migration, and poor prognostic outcomes. GJB6 is a gene encoding connexin 30 that has also recently 
been shown to regulate metastasis and chemoresistance in lung cancer[17], gastric cancer[18], renal cancer[19], glioma[20], and breast cancer[21]. The functional relevance of GJB6 in CRC, however, has yet to be defined.

CEND1 is involved in regulating CCND1[10], which is a gene specifically associated with the G1/S phase of the cell cycle, while GJB6 has been linked to the regulation of cellular proliferation via multiple pathways[22], including pathways related to CCND1. As such, we herein explored the potential prognostic relevance of CEND1 (BM88) and GJB6 (connexin30) and the correlations between these proteins in CRC patient samples.

\section{Materials And Methods}

\section{Patients and samples}

In total, samples were collected from 87 CRC (51 male, 36 female; median age: 69 years, range: $31-81$ ) patients that underwent surgical tumor resection without having undergone previous radiotherapy or chemotherapy at the first affiliated hospital of Xi'an Jiaotong university between January 2012 and December 2014. Pairs of tumor and paracancerous tissues were obtained from each patient, with all histological diagnoses having been confirmed independently by thee experienced pathologists. The Ethics Committee of the first affiliated hospital of Xi'an Jiaotong university approved the present study, which was consistent with the Declaration of Helsinki. All patients provided informed consent.

Patient clinicopathological information is compiled in Table l, with TNM staging having been conducted as per the American Joint Committee on Cancer (AJCC) criteria. Tissues were snap-frozen immediately following collection and stored at $-80^{\circ} \mathrm{C}$. Tissues used for immunohistochemical (IHC) staining were fixed for $24 \mathrm{~h}$ with $4 \%$ paraformaldehyde, dehydrated, and paraffin-embedded.

\section{Immunohistochemistry}

IHC staining was used to assess protein expression. Briefly, samples were deparaffinized, rehydrated, blocked for 10 min with $3 \% \mathrm{H}_{2} \mathrm{O}_{2}$ to suppress endogenous peroxidase activity, and then probed overnight with antibodies specific for BM88 (ab113087, 1:200, Abcam) or connexin 30 (ab199178, 1:200, Abcam) at $4^{\circ} \mathrm{C}$. Sections were then probed for $30 \mathrm{~min}$ at room temperature with biotinylated secondary antibodies, and $D A B$ was then used to detect substrates of interest, while hematoxylin was used for nuclear counterstaining.

Numbers of positive cells in 10 random fields of view were counted via microscopy, with staining intensity and percentage positive cells both being scores. Samples were then categorized into the following groups: negative (-), $\leq 5 \%$ stained cells, 0 points; weak expression (+), 1-3 points; moderate expression (++), 4-5 points; strong expression (+++), 6-7 points. Samples in the negative and weak 
staining groups (- and + ) were considered to be negative for the purposes of statistical analyses, whereas moderate and strong staining groups $(++$ and +++$)$ were considered positive.

\section{Statistical analysis}

Data were analyzed via Student's t-tests and chi-squared tests as appropriate, with Spearman correlation analyses being used to gauge the relationship between the expression of CEND1 and GJB6. SPSS v22.0 (IL, USA) was used for all statistical testing, with $\mathrm{P}<0.05$ as the significance threshold.

\section{Results}

\section{Evaluation of the relationship between CEND1 expression and CRC patient characteristics}

Pathological and demographic data corresponding to patients in the present study is compiled in Table I. An immunohistochemical staining approach was first used to evaluate CEND1 protein levels in the samples from these CRC patients. Relative to paired paracancerous tissue samples (18/87, 20.69\%), significantly more tumor samples were CEND1+ $(37 / 87,42.53 \%)(P<0.01$, Figure 1).

When the relationship between CEND1 protein levels and CRC patient clinicopathological data was evaluated, we found that CEND1 expression was significantly related to location ( $P=0.012)$, poor differentiation $(P=0.024)$, and perineural invasion $(P=0.036)$ (Table I).

\section{Evaluation of the relationship between GJB6 expression and CRC patient characteristics}

GJB6 protein levels were next assessed via IHC, revealing a higher rate of GJB6 positivity in CRC tumor tissues $(35.63 \%, 31 / 87)$ relative to paired paracancerous tissues $(18.39 \%, 16 / 87)(P<0.05$, Figure 1). The expression of GJB6 was higher in CRC patients with more advanced TNM stage $(P=0.050)$, distant metastasis $(P=0.023)$, and perineural invasion $(P=0.043)$ (Table $)$.

\section{The relationship between CEND1 and GJB6 expression in CRC}

As discussed above, CEND1 expression can promote the migration and metastasis of tumor cells through a mechanism potentially linked to the activation of GJB6. As such, we next evaluated the expression of these two proteins simultaneously in $87 \mathrm{CRC}$ patient tumor samples, separating patients into four staining categories: CEND1+/GJB6-, CEND1+/GJB6+, CEND1-/GJB6-, and CEND1-/GJB6+. This analysis revealed CEND1 and GJB6 to be strongly co-expressed, with co-expression of these markers being significant ( $r=0.534, P<0.05 ;$ Table II). In contrast, no relationship between CEND1 and GJB6 expression 
was detected in paracancerous samples $(r=0.124, P>0.05$; Table II $)$. Overall these findings suggested GJB6 and CEND1 protein levels to be correlated with one another specifically in CRC tumor tissues.

\section{The relationship between GJB6 and CEND1 levels and CRC tumor perineural invasion}

As discussed above and shown in Table $\mathbf{l}$, there was a significant relationship between perineural invasion and high expression of CEND1 (15/25, 60\%; $P=0.036)$ or GJB6 $(13 / 25,52 \% ; P=0.043)$ in CRC patients. When evaluating the relationship between combined CEND1 and GJB6 expression and perineural invasion status, we found that such invasion was observed for $40 \%$ of tumors expressing both markers (10/25). Spearman correlation analyses further confirmed that CEND1 and GJB6 co-expression was significantly correlated with perineural invasion in this patient cohort $(P<0.05$; Table III).

\section{The relationship between GJB6 and CEND1 levels and CRC patient overall survival}

We next assessed the association between CRC patient survival and the expression of CEND1 and GJB6 for all 87 patients for whom follow-up data through 2020 were available. These patients were stratified according to their CEND1 and GJB6 co-expression status (CEND1+/ GJB6-, CEND1+/ GJB6+, CEND1-/ GJB6-, and CEND1-/ GJB6+), revealing that patients co-expressing both of these proteins had signfiicantly reduced overall survival (OS) relative to the other three groups, thus suggesting that GJB2 and CEND1 are potentially valuable biomarkers of CRC patient outcomes $(P<0.05$, Figure 2).

To explore these findings in greater depth, univariate analyses of prognostic factors in these CRC patients were conducted via log-rank tests (Table IV), revealing histological grade, TNM stage, perineural invasion, CEND1 expression, GJB6 expression, and CEND1/GJB6 co-expression to all be significant predictors of CRC patient OS $(P<0.05$, Table III). Subsequent multivariate analysis confirmed that TNM stage $(P<0.01)$, perineural invasion, and CEND1+/GJB6+ expression $(P<0.05)$ were all independent predictors of CRC patient outcomes.

\section{Discussion}

Herein, we explored the expression of CEND1 and GJB6 in human CRC tumor tissue samples in order to understand the relationships between these proteins and both clinicopathological findings and patient prognosis. Overall we observed elevated levels of both CEND1 and GJB6 in tumor tissues relative to paired paracancerous samples. Increased CEND1 expression was associated with tumor location, poor differentiation, and perineural invasion, while GJB6 expression was associated with TNM stage, distant metastasis, and perineural invasion. Notably, levels of CEND1 and GJB6 were correlated with one another in CRC tumor tissues, and high levels of both of these proteins (CEND1+/GJB6+) were associated with poorer patient prognosis and a higher risk of perineural invasion. Multivariate analysis confirmed that 
both TNM stage and CEND1/GJB6 co-expression were independent risk factors associated with CRC patient survival.

Appropriately managing early-stage CRC patients can be challenging, as tumor resection and adjuvant therapies are often associated with disappointing outcomes.[1] There may therefore be a need to reevaluate patients without any evidence of metastasis prior to chemotherapy in order to consider other relevant pathological findings such as the presence of perineural invasion[8]. While lymph node metastasis and distant metastasis are well known to be associated with a poor prognosis, perineural invasion is often overlooked as a prognostic indicator, as it is not taken under consideration in the context of TNM staging. Future research is essential to understand how perineural infiltration influences overall CRC disease progression.

Perineural invasion is an important event that occurs in the context of cancer progression wherein neonatal neural cells and axons can penetrate the tumor microenvironment in response to certain cytokines in a mechanism that may mimic normal neurogenesis. CEND1 is a neuronal lineage-specific regulator of cell cycle progression and differentiation that controls the division of neuronal precursor cells and associated neuronal maturation[10]. Its relevance in the context of oncogenesis is not well understood. However, given that it regulates the cell cycle via a p53-dependent/Cyclin D1/pRb signaling pathway, it has the clear potential to influence tumor cell malignancy[11]. We herein confirmed this hypothesis, highlighting CEND1 as a promising therapeutic target in CRC that warrants further study.

Communication between cells is an essential facet of the tumor microenvironment, regulating key processes including perineural invasion-associated tumor progression. Gap junctions and the connexins that compose them are essential mediators of such cell-cell communication, facilitating metabolic and signaling exchanges between cells that can influence the progression of diseases including sensorineural hearing loss, reversible blindness, Charcot-Marie-Tooth X-linked disease, demyelinating diseases, skin diseases, arrhythmias, and cancer[23-25]. While connexin 26 and connexin 43 are often studies in the context of cancer, the role of connexin 30 (encoded by GJB6) in CRC remains to be clarified. As gap junctions can form from heteromeric or homomeric combinations of connexins, relative proportions of these proteins in cells are critical to effective cell-cell communication. Connexin 43 has previously been identified as a biomarker of positive breast cancer patient prognosis[26], whereas connexin 30 was suggested to be associated with a negative prognosis. In this study, we determined that the expression of GJB6 was associated with poor CRC patient outcomes and that this was correlated with the coexpression of CEND1. CEND1 may interact with one another through both the CCND1 pathway and other mechanisms, given that CEND1 is sensitive to intracellular calcium concentrations, which are in turn modulated by gap junction channels such as those formed by connexin 30 . However, more research is required to elucidate these mechanisms.

\section{Conclusion}


Overall, the results of the present study suggested that CEND1 and GJB6 may regulate CRC tumor cell-cell communication in the context of perineural invasion. Future studies will help to clarify the roles of connexins and BM88 in this oncogenic context, aiding in the development ofnovel efficacious treatments for this deadly disease.

\section{Declarations}

Ethical Approval and Consent to participate

This project was approved by the ethics committee of the First Affiliated Hospital, Medical College, Xi'an Jiaotong University for clinical research, and all specimens were obtained from patients with informed consent.

Human and Animal Rights

There is no experiments on human or animals.

\section{Consent for publication}

All authors have reviewed the final version of the manuscript and approved it for publication.

Availability of data and material

We declared that materials described in the manuscript, including all relevant raw data, will be freely available to any scientist wishing to use them for non-commercial purposes, without breaching participant confidentiality.

\section{Conflict of Interest}

There is no competing interest in this work.

\section{Funding}

This work was supported by Zhejiang Provincial Natural Science Foundation of China, Project number: LQ19H030012. 
Acknowledgments

This work was supported by Zhejiang Provincial Natural Science Foundation of China, Project number: LQ19H030012.

\section{References}

[1] R.L. Siegel, K.D. Miller, A. Goding Sauer, S.A. Fedewa, L.F. Butterly, J.C. Anderson, A. Cercek, R.A. Smith, A. Jemal, Colorectal cancer statistics, 2020, CA: a cancer journal for clinicians 70(3) (2020) 145-164.

[2] R.L. Siegel, K.D. Miller, H.E. Fuchs, A. Jemal, Cancer Statistics, 2021, CA: a cancer journal for clinicians 71(1) (2021).

[3] T. Hu, Y. Yao, S. Yu, H. Guo, L. Han, W. Wang, T. Tian, Y. Hao, Z. Liu, K. Nan, S. Wang, Clinicopathologic significance of CXCR4 and Nrf2 in colorectal cancer, J Biomed Res 27(4) (2013) 283-290.

[4] T.-H. Hu, Y. Yao, S. Yu, L.-L. Han, W.-J. Wang, H. Guo, T. Tian, Z.-P. Ruan, X.-M. Kang, J. Wang, S.-H. Wang, K.-J. Nan, SDF-1/CXCR4 promotes epithelial-mesenchymal transition and progression of colorectal cancer by activation of the Wnt/ $\beta$-catenin signaling pathway, Cancer letters 354(2) (2014) 417-426.

[5] I. Balciscueta, Z. Balciscueta, N. Uribe, E. García-Granero, Perineural invasion is increased in patients receiving colonic stenting as a bridge to surgery: a systematic review and meta-analysis, Tech Coloproctol (2020).

[6] T. Suzuki, K. Suwa, M. Ogawa, K. Eto, H. Kawahara, T. Fujita, M. Ikegami, K. Yanaga, Adjuvant chemotherapy for the perineural invasion of colorectal cancer, J Surg Res 199(1) (2015) 84-89.

[7] H.C. van Wyk, J. Going, P. Horgan, D.C. McMillan, The role of perineural invasion in predicting survival in patients with primary operable colorectal cancer: A systematic review, Crit Rev Oncol Hematol 112 (2017) 11-20.

[8] L.G.J. Leijssen, A.M. Dinaux, M.S. Taylor, V. Deshpande, H. Kunitake, L.G. Bordeianou, D.L. Berger, Perineural Invasion Is a Prognostic but not a Predictive Factor in Nonmetastatic Colon Cancer, Dis Colon Rectum 62(10) (2019) 1212-1221.

[9] K. Aravantinou-Fatorou, F. Ortega, D. Chroni-Tzartou, N. Antoniou, C. Poulopoulou, P.K. Politis, B. Berninger, R. Matsas, D. Thomaidou, CEND1 and NEUROGENIN2 Reprogram Mouse Astrocytes and Embryonic Fibroblasts to Induced Neural Precursors and Differentiated Neurons, Stem Cell Reports 5(3) (2015) 405-418.

[10] P.K. Politis, G. Makri, D. Thomaidou, M. Geissen, H. Rohrer, R. Matsas, BM88/CEND1 coordinates cell cycle exit and differentiation of neuronal precursors, Proceedings of the National Academy of Sciences of 
the United States of America 104(45) (2007) 17861-17866.

[11] K. Tsioras, F. Papastefanaki, P.K. Politis, R. Matsas, M. Gaitanou, Functional Interactions between BM88/Cend1, Ran-binding protein M and Dyrk1B kinase affect cyclin D1 levels and cell cycle progression/exit in mouse neuroblastoma cells, PloS one 8(11) (2013) e82172.

[12] P.K. Politis, S. Akrivou, C. Hurel, O. Papadodima, R. Matsas, BM88/Cend1 is involved in histone deacetylase inhibition-mediated growth arrest and differentiation of neuroblastoma cells, Febs Lett 582(5) (2008) 741-748.

[13] T. Aasen, I. Sansano, M.A. Montero, C. Romagosa, J. Temprana-Salvador, A. Martinez-Marti, T. Moline, J. Hernandez-Losa, S. Ramon y Cajal, Insight into the Role and Regulation of Gap Junction Genes in Lung Cancer and Identification of Nuclear Cx43 as a Putative Biomarker of Poor Prognosis, Cancers (Basel) 11(3) (2019).

[14] R.J. Ruch, Connexin43 Suppresses Lung Cancer Stem Cells, Cancers (Basel) 11(2) (2019).

[15] J. Yang, G. Qin, M. Luo, J. Chen, Q. Zhang, L. Li, L. Pan, S. Qin, Reciprocal positive regulation between Cx26 and PI3K/Akt pathway confers acquired gefitinib resistance in NSCLC cells via GJIC-independent induction of EMT, Cell Death Dis 6 (2015) e1829.

[16] S.G. Zeng, X. Lin, J.C. Liu, J. Zhou, Hypoxiainduced internalization of connexin 26 and connexin 43 in pulmonary epithelial cells is involved in the occurrence of nonsmall cell lung cancer via the P53/MDM2 signaling pathway, Int J Oncol 55(4) (2019) 845-859.

[17] I. Donner, R. Katainen, L.J. Sipilä, M. Aavikko, E. Pukkala, L.A. Aaltonen, Germline mutations in young non-smoking women with lung adenocarcinoma, Lung Cancer 122 (2018) 76-82.

[18] H.J. Son, C.H. An, N.J. Yoo, S.H. Lee, Tight Junction-Related CLDN5 and CLDN6 Genes, and Gap Junction-Related GJB6 and GJB7 Genes Are Somatically Mutated in Gastric and Colorectal Cancers, Pathol Oncol Res 26(3) (2020) 1983-1987.

[19] J. Luo, Y. Xie, Y. Zheng, C. Wang, F. Qi, J. Hu, Y. Xu, Comprehensive insights on pivotal prognostic signature involved in clear cell renal cell carcinoma microenvironment using the ESTIMATE algorithm, Cancer medicine 9(12) (2020) 4310-4323.

[20] M. Artesi, J. Kroonen, M. Bredel, M. Nguyen-Khac, M. Deprez, L. Schoysman, C. Poulet, A. Chakravarti, H. Kim, D. Scholtens, T. Seute, B. Rogister, V. Bours, P.A. Robe, Connexin 30 expression inhibits growth of human malignant gliomas but protects them against radiation therapy, Neuro Oncol 17(3) (2015) 392406.

[21] I. Teleki, A.M. Szasz, M.E. Maros, B. Gyorffy, J. Kulka, N. Meggyeshazi, G. Kiszner, P. Balla, A. Samu, T. Krenacs, Correlations of differentially expressed gap junction connexins Cx26, Cx30, Cx32, Cx43 and Cx46 with breast cancer progression and prognosis, PloS one 9(11) (2014) e112541. 
[22] J.J. Kelly, Q. Shao, D.J. Jagger, D.W. Laird, Cx30 exhibits unique characteristics including a long halflife when assembled into gap junctions, J Cell Sci 128(21) (2015) 3947-3960.

[23] M. Hernández-Guerra, A. Hadjihambi, R. Jalan, Gap junctions in liver disease: Implications for pathogenesis and therapy, J Hepatol 70(4) (2019) 759-772.

[24] D.W. Laird, P.D. Lampe, Therapeutic strategies targeting connexins, Nat Rev Drug Discov 17(12) (2018) 905-921.

[25] T. Aasen, M. Mesnil, C.C. Naus, P.D. Lampe, D.W. Laird, Gap junctions and cancer: communicating for 50 years, Nature reviews. Cancer 16(12) (2016) 775-788.

[26] S. Fostok, M. El-Sibai, D. Bazzoun, S. Lelievre, R. Talhouk, Connexin 43 Loss Triggers Cell Cycle Entry and Invasion in Non-Neoplastic Breast Epithelium: A Role for Noncanonical Wnt Signaling, Cancers (Basel) 11(3) (2019).

\section{Tables}

Table / Association of CEND1 and GJB6 expressions with clinicopathological characteristics of CRC patients 


\begin{tabular}{|c|c|c|c|c|c|c|c|}
\hline & & CEND1 & & & GJB6 & & \\
\hline & $n(\%)$ & Positive & Negative & $P$ & Positive & Negative & $P$ \\
\hline \multicolumn{8}{|l|}{ Age } \\
\hline$<60 y$ & $\begin{array}{l}35 \\
(40.23)\end{array}$ & 15 & 20 & 0.959 & 16 & 19 & 0.107 \\
\hline$\geq 60 y$ & $\begin{array}{l}52 \\
(59.77)\end{array}$ & 22 & 30 & & 15 & 37 & \\
\hline \multicolumn{8}{|l|}{ Gender } \\
\hline Male & $\begin{array}{l}47 \\
(54.02)\end{array}$ & 18 & 29 & 0.387 & 17 & 30 & 0.910 \\
\hline Female & $\begin{array}{l}40 \\
(45.98)\end{array}$ & 19 & 21 & & 14 & 26 & \\
\hline \multicolumn{8}{|l|}{ Tumor Location } \\
\hline Colon & $\begin{array}{l}51 \\
(58.62)\end{array}$ & 16 & 35 & $0.012^{\star}$ & 14 & 37 & 0.058 \\
\hline Rectum & $\begin{array}{l}36 \\
(41.38)\end{array}$ & 21 & 15 & & 17 & 19 & \\
\hline \multicolumn{8}{|l|}{ CEA } \\
\hline$<3.4 \mathrm{ng} / \mathrm{mL}$ & $\begin{array}{l}30 \\
(34.48)\end{array}$ & 15 & 15 & 0.306 & 11 & 19 & 0.884 \\
\hline$\geq 3.4 \mathrm{ng} / \mathrm{mL}$ & $\begin{array}{l}57 \\
(65.52)\end{array}$ & 22 & 35 & & 20 & 37 & \\
\hline \multicolumn{8}{|l|}{ Tumor size } \\
\hline$<5 \mathrm{~cm}$ & $\begin{array}{l}36 \\
(41.38)\end{array}$ & 11 & 25 & 0.058 & 10 & 26 & 0.199 \\
\hline$\geq 5 \mathrm{~cm}$ & $\begin{array}{l}51 \\
(58.62)\end{array}$ & 26 & 25 & & 21 & 30 & \\
\hline \multicolumn{8}{|c|}{ Histological grade } \\
\hline Well/moderate & $\begin{array}{l}38 \\
(43.68)\end{array}$ & 11 & 27 & $0.024^{\star}$ & 16 & 22 & 0.267 \\
\hline Poor & $\begin{array}{l}49 \\
(56.32)\end{array}$ & 26 & 23 & & 15 & 34 & \\
\hline \multicolumn{8}{|l|}{ TNM stage } \\
\hline$I+I I$ & $\begin{array}{l}34 \\
(39.08)\end{array}$ & 16 & 18 & 0.494 & 8 & 26 & $0.050 *$ \\
\hline III + IV & 53 & 21 & 32 & & 23 & 30 & \\
\hline
\end{tabular}


Lymph node metastasis

\begin{tabular}{|c|c|c|c|c|c|c|c|}
\hline Positive & $\begin{array}{l}33 \\
(37.93)\end{array}$ & 13 & 20 & 0.644 & 11 & 22 & 0.726 \\
\hline Negative & $\begin{array}{l}54 \\
(62.07)\end{array}$ & 24 & 30 & & 20 & 34 & \\
\hline \multicolumn{8}{|c|}{$\begin{array}{l}\text { Distant } \\
\text { metastasis }\end{array}$} \\
\hline Positive & $\begin{array}{l}27 \\
(31.03)\end{array}$ & 8 & 19 & 0.103 & 14 & 13 & $0.034^{*}$ \\
\hline Negative & $\begin{array}{l}60 \\
(68.97)\end{array}$ & 29 & 31 & & 17 & 43 & \\
\hline \multicolumn{8}{|l|}{$\begin{array}{l}\text { perineural } \\
\text { invasion }\end{array}$} \\
\hline Positive & $\begin{array}{l}25 \\
(28.73)\end{array}$ & 15 & 10 & $0.036^{*}$ & 13 & 12 & $0.043 *$ \\
\hline Negative & $\begin{array}{l}62 \\
(71.27)\end{array}$ & 22 & 40 & & 18 & 44 & \\
\hline
\end{tabular}

* $P<0.05$

Table I/ Correlation between CEND1 and GJB6 expression in CRC and normal tissues

\begin{tabular}{|lllll|}
\hline GJB6 & \multicolumn{2}{l}{ CEND1 } & \multicolumn{1}{c|}{$r$} & $P$ \\
\cline { 2 - 4 } & Positive & Negative & & \\
\cline { 1 - 4 } Tumor & & & & \\
\hline Positive & $18(\%)$ & $13(\%)$ & 0.534 & $<0.05$ \\
\hline Negative & $19(\%)$ & $37(\%)$ & & \\
\hline Normal & & & & \\
\hline Positive & $5(\%)$ & $11(\%)$ & 0.124 & $>0.05$ \\
\hline Negative & $13(\%)$ & $58(\%)$ & & \\
\hline
\end{tabular}

Table III Correlation of combined both expression of CEND1 and GJB6 with perineural invasion in CRC 


\begin{tabular}{|llll|}
\hline CEND1 and GJB6 & \multicolumn{2}{l|}{ perineural invasion } & \multirow{2}{*}{$P$} \\
\cline { 2 - 3 } & Positive & Negative & \\
\hline Both positive & $10(38.16 \%)$ & $41(9.21 \%)$ & 0.025 \\
\hline Others & $15(25.00 \%)$ & $21(27.63 \%)$ & \\
\hline
\end{tabular}

Table VI. Univariate analysis of survival data

\begin{tabular}{|ll|}
\hline Clinicopathological characteristics & P-value \\
\hline Histological grade, well/moderate compared with poor & $0.018^{\star}$ \\
\hline TNM stage, I + II compared with III + IV & $0.020^{\star}$ \\
\hline Perineural invasion, positive compared with negative & $0.040^{\star}$ \\
\hline CEND1, positive compared with negative & $0.038^{\star}$ \\
\hline GJB6, positive compared with negative & $0.041^{\star}$ \\
\hline Co-expression of CEND1 and GJB6, CEND1+/GJB6+ compared with others & $0.031^{\star}$ \\
\hline
\end{tabular}

* $P<0.05$

Figures 
A

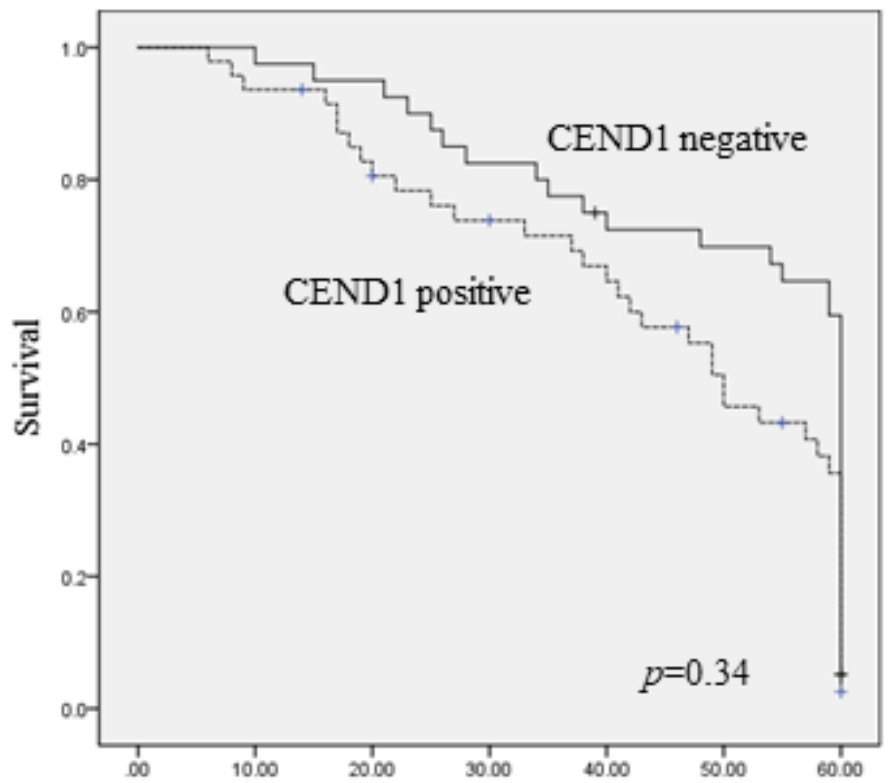

$\mathrm{C}$

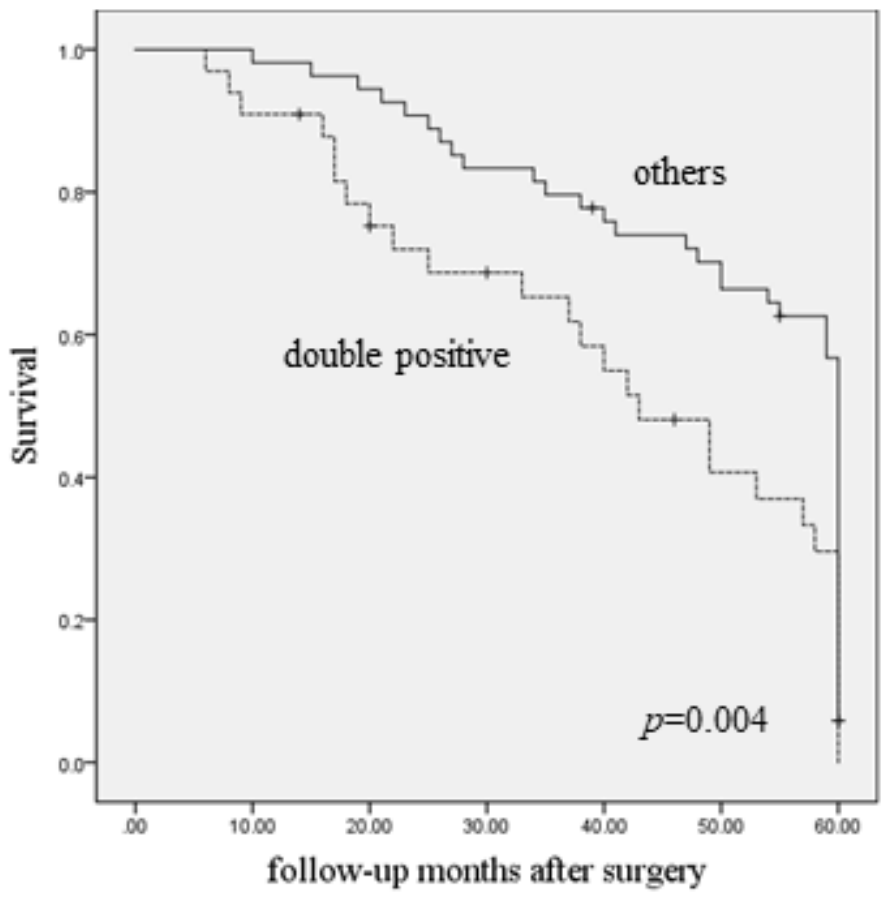

B

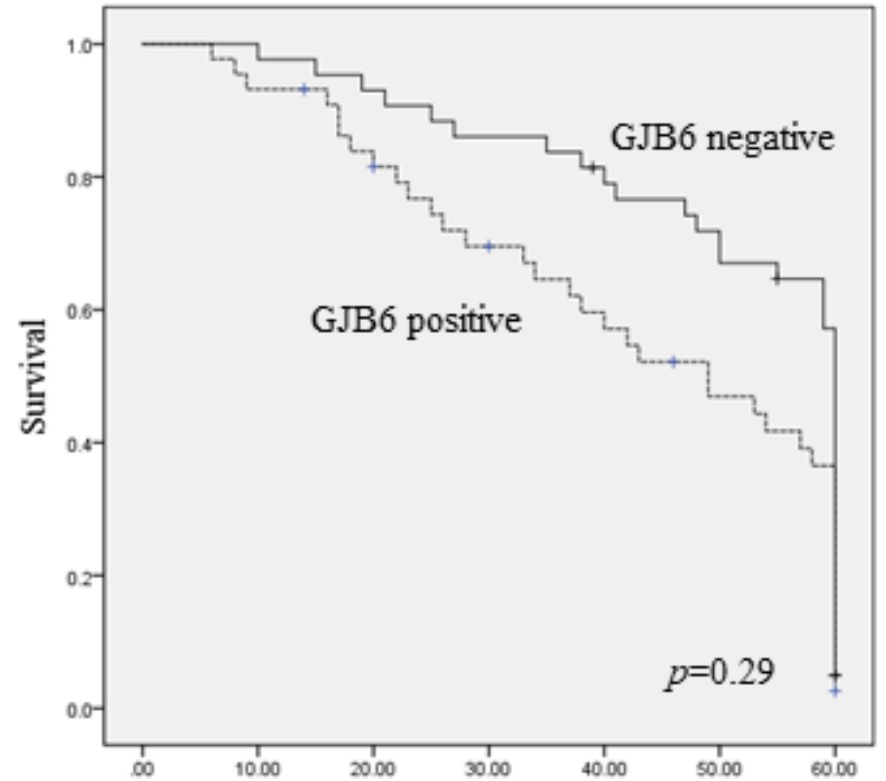

$\mathrm{D}$

follow-up months after surgery

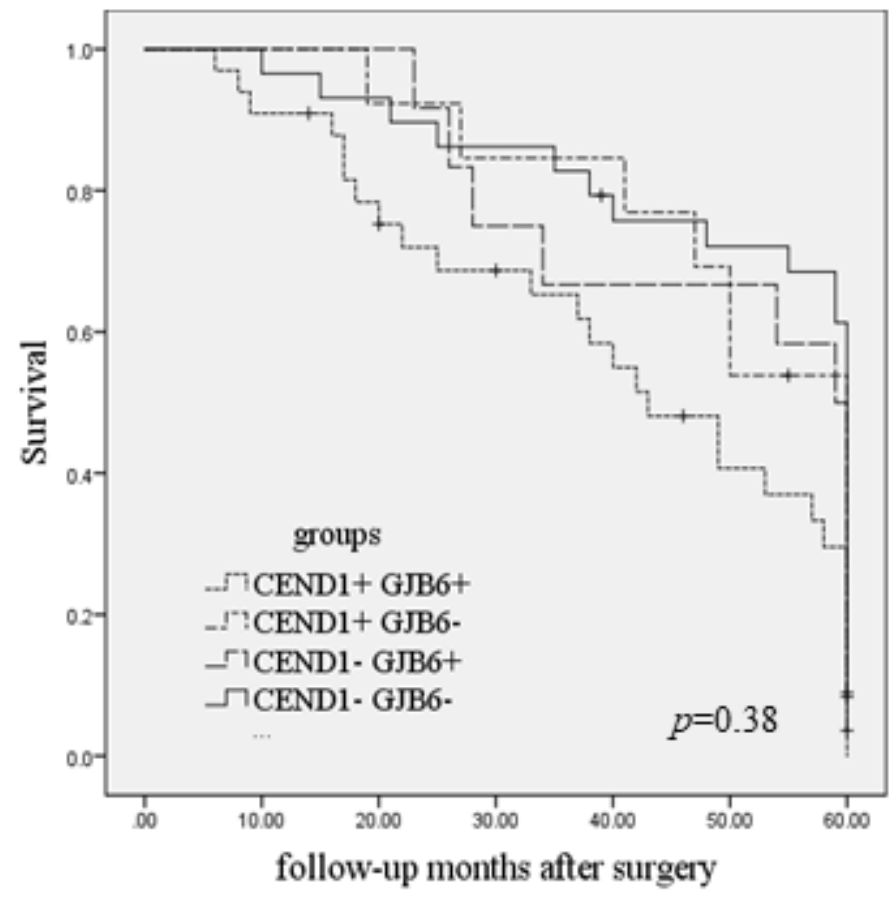

Figure 1

Immunohistochemistry of CEND1 and GJB6 in colorectal cancer (CRC). A: High expression of CEND1 in tumor tissues ( $\times 200)$; B: High expression of CEND1 in Tumor tissues $(\times 200)$; C: Low expression of GJB6 in CRC tissues (×200); D: Low expression of GJB6 in adjacent non-tumors tissues $(\times 200)$. 
A

$\mathrm{C}$
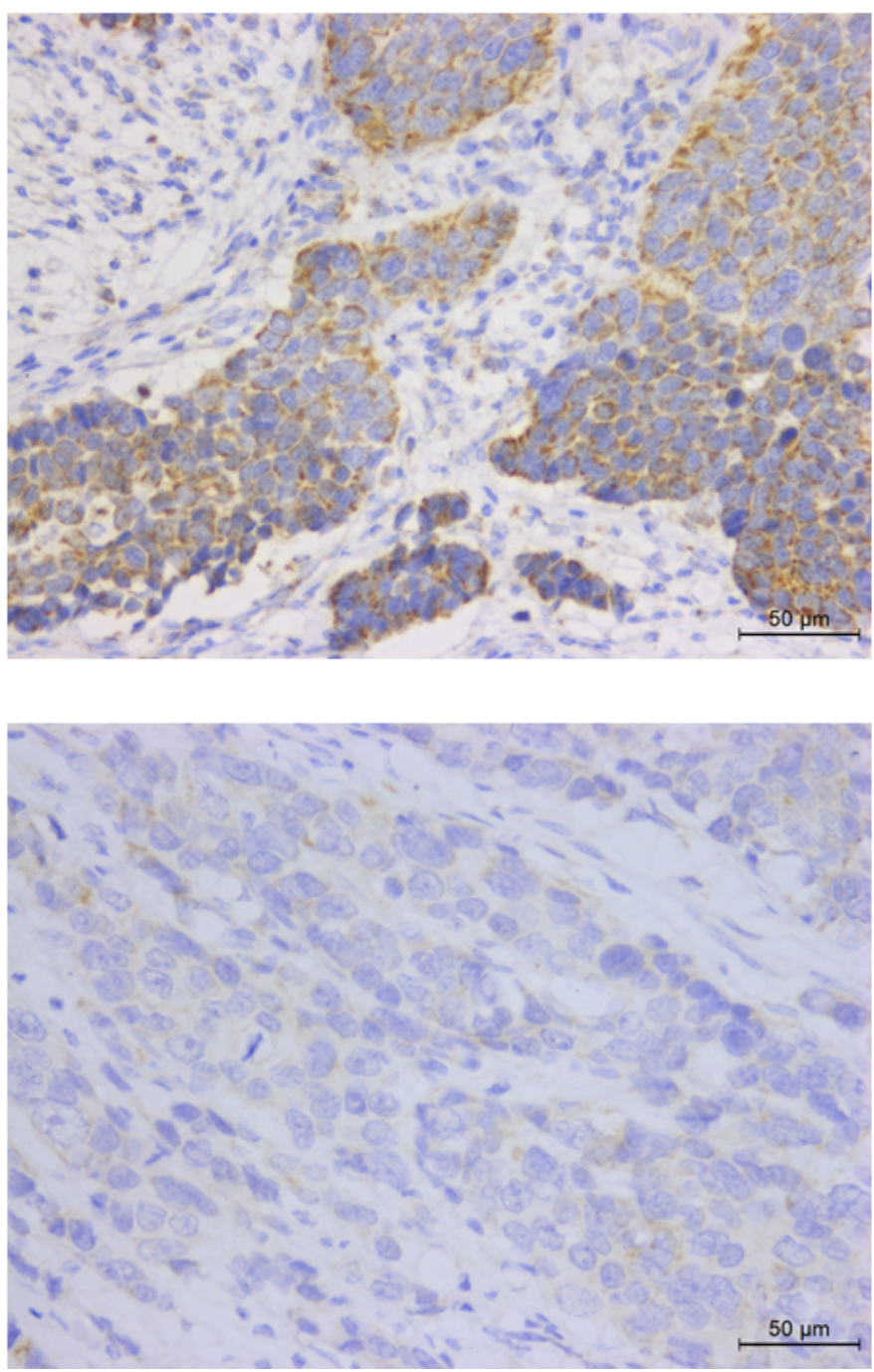

$\mathrm{B}$

$\mathrm{D}$
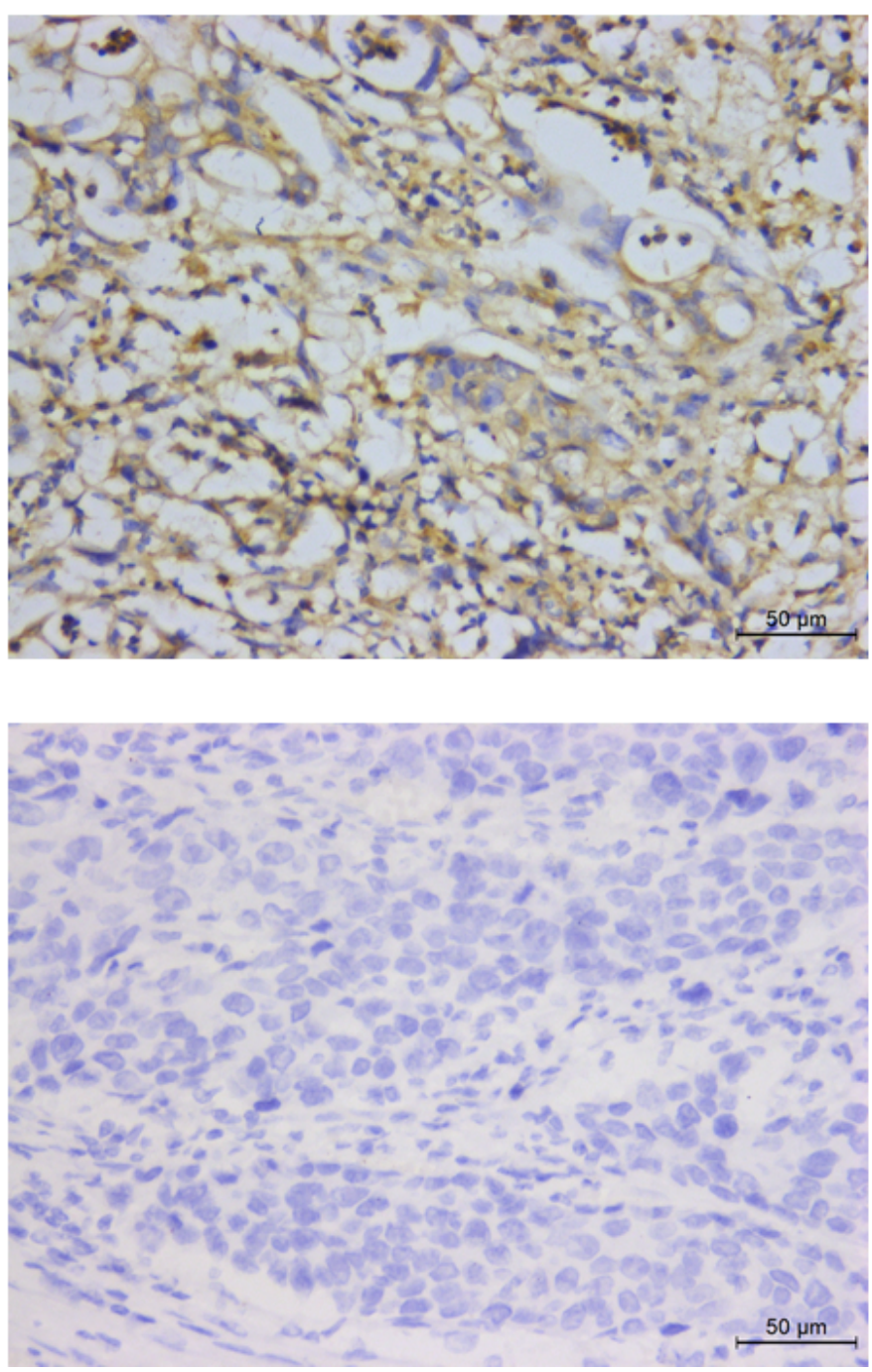

Figure 2

Kaplan-Meier survival curve analyses of the association between CEND1 and GJB6 expression and overall survival of GC patients using the log rank test. (A) CEND1 expression; (B) GJB6 expression; (C) CEND1+/GJB6+ compared with other expression combinations; (D) comparison of CEND1+/GJB6+, CEND1+/GJB6-, CEND1-/GJB6+ and CEND1-/GJB6-. 